\title{
Changes in the National Cancer Institute's R01 workforce: growth, aging, retention, and policy implications
}

\author{
Melissa D. Antman, Roman Gorelik, Amy Kennedy, Grace F. Liou, Eddie N. Billingslea, James G. Corrigan, and L. Michelle Bennett \\ Center for Research Strategy, National Cancer Institute, NIH, Bethesda, Maryland, USA.

\begin{abstract}
Scientific progress and discovery of preventions and cures for life-threatening diseases depend on the vitality of the biomedical research workforce. We analyzed the workforce of cancer researchers applying for and receiving R01 awards from the National Cancer Institute (NCI) from fiscal years 1990 to 2016, the last year prior to implementation of the Next Ceneration Researchers Initiative. Here we report that the NCI R01 Principal Investigator (PI) workforce expanded 1.4-fold and aged over this time frame. We tracked 9 age groups and found that the number of PIs in the 3 oldest groups increased dramatically, in contrast with the younger groups. Sustained increases in the number of funded older Pls stemmed from increases in the number of older PIs submitting applications, rather than higher funding rates for older PIs. The decline in the number of funded younger PIs was driven in part by (a) a marked increase in time from PhD degree to first R01 application and award, as well as (b) a decrease in retention of Pls in the funded R01 workforce beyond their first R01 award. The NCI is using these and other analyses to inform strategies and policies for attracting, supporting, and retaining meritorious early-career researchers.
\end{abstract}

\section{Introduction}

As the leader of the National Cancer Program, the mission of the National Cancer Institute (NCI) is to lead, conduct, and support cancer research across the nation to advance scientific knowledge and help all people live longer, healthier lives (1). The NCI is the largest institute of the National Institutes of Health (NIH), with a budget of $\$ 6.4$ billion in fiscal year (FY) 2020, of which $\$ 1.6$ billion was spent on R01 grants. These substantial research grants have been the most common funding source for launching independent research careers. The success of the National Cancer Program depends on attracting new researchers and sustaining them in the cancer research workforce, in particular, as Principal Investigators (PIs) on NCI R01 awards (see Table 1 for a complete list of definitions).

Many studies have reported the aging of the scientific workforce (2-7), including the NIH workforce, which, over the last 30 years, has experienced increases in both the average age of PIs and the average age at which they receive their first substantial NIH grant (8-10). A study of US-trained scientists and engineers with research doctorate degrees showed that the main contributing factors to workforce aging included the aging of the large cohort of baby boomers and the declines in retirement rates of faculty following the 1994 repeal of the mandatory retirement age requirement (11). The same study showed that this scientific workforce aged considerably faster than the US workforce as a whole.

Here we seek to understand how the age and career stage of PIs applying for and receiving NCI R01 awards changed over

Conflict of interest: The authors have declared that no conflict of interest exists. Copyright: (c) 2021, American Society for Clinical Investigation.

Reference information: J Clin Invest. 2021;131(7):e146925.

https://doi.org/10.1172/JCl146925. time. We first describe the aging of the NCI workforce over a 26-year period. In describing workforce aging, we use terms such as "younger" and "older" investigator to denote chronological age. Separately, we also examine career stage (Figure 1), for which we use terms such as "early-career," "early-stage," and "new" investigator. For example, a new investigator is not necessarily young. Career stage is distinct from chronological age, although early-career investigators as a group tend to be younger than established investigators.

The aging of the scientific workforce may have several consequences. On the one hand, high-performing older scientists may be able to extend their research productivity. On the other hand, workforce aging may create a high barrier to entry for incoming early-career PIs, who are competing for fewer available positions, since these positions are occupied by non-retiring established researchers (12). As a result, talented young researchers may be lost to other fields and career opportunities.

To encourage PIs to apply earlier in their careers for NIH R01 support, the NIH implemented the Early Stage Investigator (ESI) policy in 2009, which provided special consideration both in review and for funding of R01 applications from early-career scientists starting their independent research programs. The NIH defines ESIs as PI applicants who are within 10 years of their terminal research degree (or clinical training) and who have not yet competed successfully for a substantial NIH research grant (see Table 1 for complete definitions). ESIs are a subset of $\mathrm{New}$ Investigators (NIs), defined as PIs who have not competed successfully for a substantial NIH award. The ESI policy served as a foundation for the NIH's Next Generation Researchers Initiative (NGRI), launched in 2017 and called for in the 21st Century Cures Act to develop policies and programs "that are focused on promoting and providing opportunities for new researchers 


\section{Table 1. Definitions}

Competing application

Early Stage Investigator (ESI)

Established Investigator (EI)

Funding rate

Multiple Principal Investigator (MPI) application/award

New Investigator (NI)

Non-competing continuation application/award

Principal Investigator (PI)

R01 equivalent
New or renewal application that must undergo initial peer review

A Principal Investigator who has completed his or her terminal research degree or end of postgraduate clinical training, whichever date is later, within the past 10 years and who has not previously competed successfully as a PI for a substantial $\mathrm{NIH}$ independent research award

A Principal Investigator who has previously competed successfully as a PI for a substantial NIH independent research award Percentage of applicant Pls who receive an award in a fiscal year

An application or award with multiple individuals designated as Pls who share the authority and responsibility for leading and directing the project

A Principal Investigator who has not previously competed successfully as a PI for a substantial NIH independent research award

A financial assistance request (in the form of an application or progress report) or resulting award for a subsequent budget period within a previously approved project period for which a recipient does not have to compete with other applicants The individual(s) designated by the applicant organization to have the appropriate level of authority and responsibility to direct the project or program to be supported by the award

R01 equivalents include substantial NIH research grants like the R01 that are or have historically been used as funding vehicles to launch an independent research career. The collection of awards considered R01 equivalents changes over time, reflecting changes in the types of grants being offered by the $\mathrm{NIH}$. In FY 2016, and in these analyses, R01 equivalents included the grant activity codes R01, R23, R29, R37, DP2, and RF1.

Sequestration A statutory requirement for the NIH to cut $5 \%$ of its FY 2013 budget

Success rate and earlier research independence" (13). The aging of the NIH workforce and the NGRI prompted us to conduct an analysis of characteristics of NCI PIs.

The analysis presented in this paper focuses on PIs applying for and receiving NCI R01 awards. We based these analyses on R01 PIs for several reasons: (a) the NGRI and ESI policies focus on R01s, (b) the R01 is the most awarded research project grant at the NCI and at the NIH, (c) the funding amount and duration of a typical R01 are considered substantial funding enabling support of a single PI's research program, and (d) the R01 is used similarly across the NIH. In this analysis, we also include R01-equivalent awards, as defined by the NIH (Table 1), which comprise the NIH activity codes R23, R29, R37, DP2, and RF1. For the sake of brevity, hereafter we refer to R01s and R01 equivalents collectively as "R01s."
An NCI R01 follows a particular life cycle, which influences a given PI's timing in applying for new grants and in competing for renewal. NCI R01s are typically awarded for 5 consecutive years. The first year of the R01 is called a new competing (Type 1) award, and the remaining 4 years are non-competing renewal (Type 5) awards. PIs have the option to submit a competing renewal (Type 2) application of their original R01 award, which, if awarded, would start at the end of their initial R01 award period. PIs with funded R01s are eligible to compete for multiple renewals.

In this paper, we report a dramatic increase in the age of $\mathrm{NCI}$ R01 awardees from FY 1990 to FY 2016. We show that, over the same time frame, the percentage of young investigators applying for and receiving awards declined substantially. We also describe and quantify changes in timing of and age at specific milestones in a PI's career, such as the receipt of a terminal degree, submission of a first NIH R01 application, and receipt of a first R01 award. We focus on how and why the workforce aged, in terms of both chronological age and career stage. This work examines how multiple factors affected the demographics of the NCI workforce over time.

\section{Results}

Trends in age distribution of NCI RO1 PIs. To examine changes in age distribution of NCI-funded R01 PIs from FY 1990 to FY 2016, for each fiscal year, we determined the number of R01 awardees on all awards, competing and noncompeting, for each of the 9 age groups. Analyzing the workforce using 5-year groups enabled us to construct a high-resolution view of changes in the distribution of age over time. Previously

Figure 1. Schematic of career stages. 


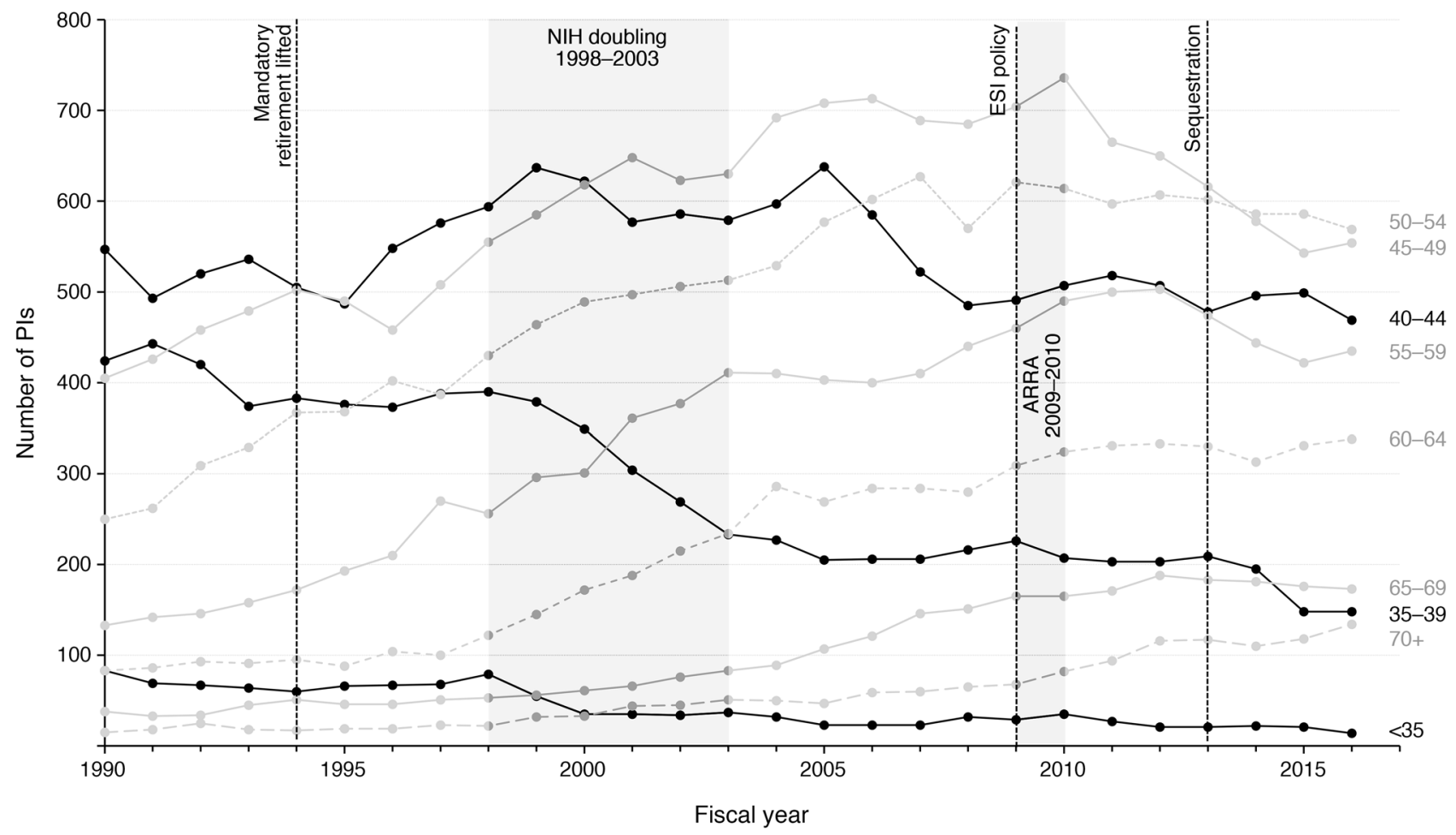

Figure 2. Age distribution of NCI Principal Investigators from FY 1990 to FY 2016. The number of PIs who hold R01-equivalent grants (competing and noncompeting) in any given year is shown in 5-year groups, starting at under age 35 and ending with over age 70 . This graph does not include the approximately $5 \%$ of all NCI PIs whose age is not available. Vertical lines show events that may have affected NIH funding and application behavior, including the lifting of mandatory retirement age for faculty, the doubling of the NIH budget, the American Recovery and Reinvestment Act (ARRA), the implementation of the NIH Early Stage Investigator (ESI) policy, and the sequestration.

reported analyses of the NIH workforce used 15-year age groups (14-18), and reached somewhat different conclusions. In our analysis, for each age group we display the number of awarded PIs over time (Figure 2) and show the proportion for selected fiscal years (Table 2).

Over the entire time frame, both the number and the proportion of total R01 awardees increased for every age group except for the 3 youngest $(<35,35-39$, and $40-44)$. In addition, the $45-49$ age group increased from 1990 to 2010, but it decreased substantially between 2010 and 2016. The numbers of awardees for the under 35 group and the 35-39 group both decreased. The size of the 40-44 age group fluctuated over time with no net change. Overall, awardees aged 44 and younger decreased substantially as a proportion of the total number of awardees, from $47 \%$ in FY 1990 to $21 \%$ in FY 2016 (Table 2). Specifically, the percentage of awardees under 35 decreased from $4 \%$ to $0.5 \%$, aged $35-39$ decreased from $19 \%$ to $5 \%$, and aged $40-44$ decreased from $24 \%$ to $15 \%$. In contrast, the number of PIs in the 45-49 age group increased 1.4-fold, and the number of PIs in the 3 next older age groups, encompassing PIs aged 50 through 64 years, grew at least 2-fold. Notably, the older age groups (65-69 and 70+) emerged during this time frame and continued to increase in number and proportion. The total number of awardees 65 and older increased approximately 6-fold since FY 1994. The proportion of awardees 65 and older remains only at $10 \%$.

The NCI R01 PI workforce has simultaneously grown older and larger. The observed decreases in the number of funded PIs in the younger groups and the concurrent increases in the older age groups indicate that the NCI R01 PI workforce has aged. The total number of PIs holding NCI R01 awards increased 1.4-fold during the overall time frame, from 2258 in FY 1990 to 3095 in FY 2016, peaking at 3428 PIs in FY 2010, owing in part to the American Recovery and Reinvestment Act (ARRA).

Age and funding rates of competing applicants and awardees. The aging of the NCI R01 PI workforce led us to hypothesize that either (a) older PIs were more successful at competing for funding than younger PIs, or (b) increasing numbers of older PIs were submitting applications relative to younger PIs. To test the first hypothesis, we determined the funding rates for competing applicants among the 9 age groups for selected years: FYs 1990, 2000, 2010, and 2016 (Table 3; for definition of "funding rate" see Table 1). The funding rates for NCI R01 awards decreased over time, consistent with what has been shown for the NIH as a whole (8), and the decrease was similar across all age groups. Importantly, in any given fiscal year of the 4 shown here, the funding rates were similar for all age groups, with no statistically significant differences observed, indicating that young PIs were as successful in competing for NCI R01s as those in the other age groups.

To test the second hypothesis, that increasing numbers of older PIs were submitting applications relative to younger PIs, we counted the number of applicants in the 9 age groups over time (Table 3). The total number of applicants more than doubled between FY 1990 and FY 2016, increasing in all age groups except the 2 youngest. The number of 35- to 39-year-old applicants remained flat, whereas the number of applicants under 35, while already small in FY 1990, 
Table 2. Distribution of NCI R01 Pls showing number and proportion in each age group in selected fiscal years

\begin{tabular}{|c|c|c|c|c|c|}
\hline Age group (years) & FY 1990 & FY 1994 & FY 2000 & FY 2010 & FY 2016 \\
\hline$<35$ & $83(4 \%)$ & $60(3 \%)$ & $35(1 \%)$ & $35(10 \%)$ & $14(<1 \%)$ \\
\hline $35-39$ & $424(19 \%)$ & $383(17 \%)$ & $349(12 \%)$ & $207(6 \%)$ & $148(5 \%)$ \\
\hline $40-44$ & $547(24 \%)$ & $505(22 \%)$ & $622(22 \%)$ & $507(15 \%)$ & $469(15 \%)$ \\
\hline $50-54$ & $250(11 \%)$ & $367(16 \%)$ & $489(17 \%)$ & $614(18 \%)$ & $569(18 \%)$ \\
\hline $55-59$ & $133(6 \%)$ & $172(7 \%)$ & $301(11 \%)$ & $490(14 \%)$ & $435(14 \%)$ \\
\hline $70+$ & $15(<1 \%)$ & $17(<1 \%)$ & $33(1 \%)$ & $82(2 \%)$ & $134(4 \%)$ \\
\hline Unknown age & $280(12 \%)$ & $169(7 \%)$ & $195(7 \%)$ & $268(8 \%)$ & $261(8 \%)$ \\
\hline Total & 2258 & 2321 & 2875 & 3428 & 3095 \\
\hline
\end{tabular}

The total number of PIs peaked in FY 2010, likely owing in part to the increased budget from the American Recovery and Reinvestment Act. The percentage of PIs in the unknown age category remained fairly constant over this time frame, which allows us to compare the trends between age groups with more certainty.

decreased by approximately two-thirds by FY 2016. In contrast, the number of applicants in the older age groups increased substantially: 4-fold for the 50-54 group, 6-fold for the 55-59 group, 7-fold for the 60-64 group, and 8-fold for the 65-69 group. Thus, the proportion of applicants in the youngest age groups declined as a result of manyfold increases in numbers of older applicants and as a result of lack of growth in numbers of applicants in the 2 youngest groups.

Career stage and age at first NCI RO1 award. Given the decrease in number of young applicants and awardees, we hypothesized that over time PIs were taking longer to enter the NCI R01 workforce. To test this hypothesis, we examined the time elapsed from receipt of $\mathrm{PhD}$ degree to first R01 award. This duration doubled, from a median of 6 years in FY 1990 to 12 years in FY 2016 (Figure 3). Increases in time to first R01 award started to stabilize in FY 2005, similar to what has been shown for the NIH as a whole (19). Similar trends held for PIs with MDs and MD/PhDs, although the actual times until first R01 award were longer because of intervening clinical training, and the data are complicated by variability in time committed to clinical training (Supplemental Figure 1; supplemental material available online with this article; https://doi. org/10.1172/JCI146925DS1).

The time from degree to first R01 award can encompass multiple career stages, including postdoctoral training, academic job search, lab startup, writing and submission of the first R01 application, and time for application review and funding. For some NIs this period could also include time spent in careers outside academic research, as well as time conducting research funded by small NIH grants or nonNIH grants. Although we did not have data on each of these stages individually, we had access to year of degree, year of first R01 application, and year of first R01 award. To further understand what contributed most to the increase in time from degree to award, we analyzed 2 components: (a) time from degree to submission of first R01 application and (b) time from submission of first R01 application to first R01 award. While both components increased over time, approximately two-thirds of the observed increase in time from degree to award was due to the time period between $\mathrm{PhD}$ and first $\mathrm{R} 01$.

Table 3. Numbers of applicants and awardees and funding rates for PIs with competing NCI R01-equivalent applications in FYs 1990, 2000, 2010, and 2016 by 5-year age group

\begin{tabular}{|c|c|c|c|c|c|c|c|c|c|c|c|c|}
\hline $\begin{array}{l}\text { Age group } \\
\text { (years) }\end{array}$ & \multicolumn{3}{|c|}{ FY 1990} & \multicolumn{3}{|c|}{ FY 2000} & \multicolumn{3}{|c|}{ FY 2010} & \multicolumn{3}{|c|}{ FY 2016} \\
\hline $35-39$ & 435 & 116 & $27 \%$ & 367 & 108 & $29 \%$ & 345 & 80 & $23 \%$ & 351 & 59 & $17 \%$ \\
\hline $40-44$ & 432 & 127 & $29 \%$ & 590 & 160 & $27 \%$ & 701 & 146 & $21 \%$ & 781 & 127 & $16 \%$ \\
\hline $45-49$ & 347 & 105 & $30 \%$ & 560 & 181 & $32 \%$ & 868 & 188 & $22 \%$ & 848 & 167 & $20 \%$ \\
\hline $60-64$ & 65 & 17 & $26 \%$ & 144 & 47 & $33 \%$ & 313 & 79 & $25 \%$ & 464 & 80 & $17 \%$ \\
\hline $65-69$ & 31 & Too few & - & 42 & 13 & $31 \%$ & 158 & 31 & $20 \%$ & 249 & 48 & $19 \%$ \\
\hline $70+$ & Too few & Too few & - & 47 & 12 & $26 \%$ & 85 & 18 & $21 \%$ & 167 & 33 & $20 \%$ \\
\hline Age unknown & 522 & 74 & $14 \%$ & 239 & 54 & $23 \%$ & 390 & 75 & $19 \%$ & 522 & 66 & $13 \%$ \\
\hline
\end{tabular}

"Too few" indicates fewer than 12 . Within each fiscal year, funding rates between age groups were not statistically different based on $\chi^{2}$ test. 


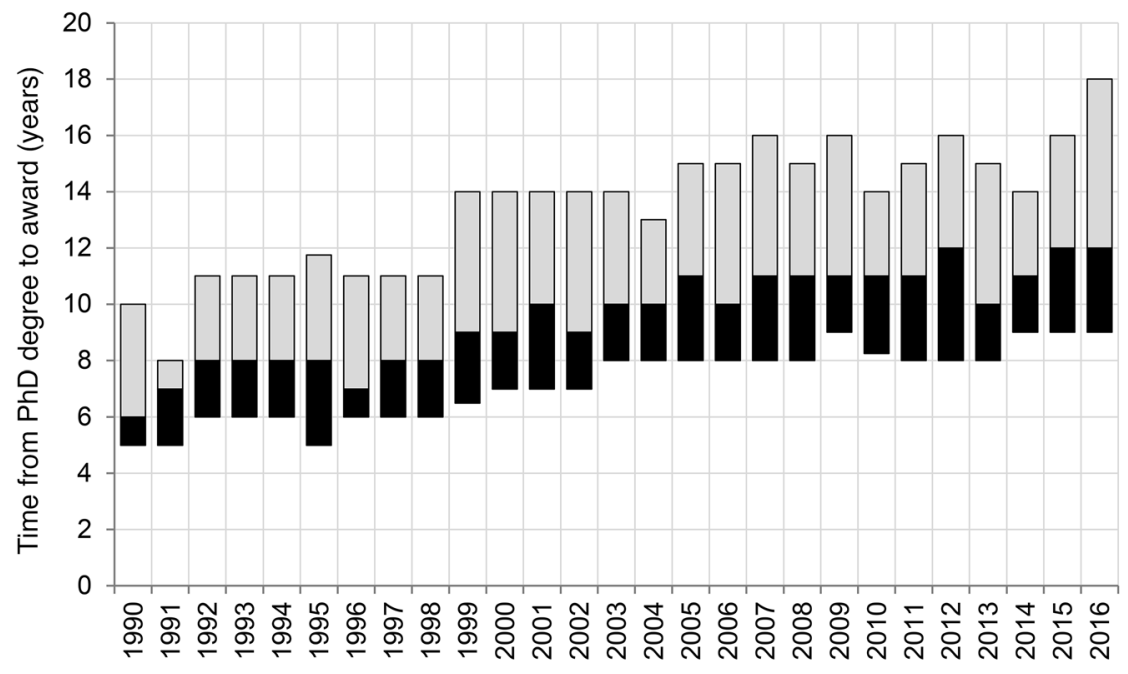

Fiscal year of first R01 (or R01-equivalent) award

Figure 3. Years from PhD degree to first R01-equivalent award. The box plots show an overall increasing trend in time (median, in years) from degree to first R01-equivalent award from FY 1990 to FY 2016. The middle line denotes the median years, with the first (black) and third (gray) quartiles marked.

We expected that the increase in time from degree to award would be associated with an increase in age of PIs at first R01, provided that the age at $\mathrm{PhD}$ did not decrease. Age at receipt of $\mathrm{PhD}$ degree for NIs increased only slightly over this time frame (Supplemental Figure 2). However, the age of PIs with PhDs at the time of first NCI R01 award increased from an average of 36.2 years in FY 1990 to 44.9 years in FY 2016. Likewise, the median age increased from 36 to 43 years (Supplemental Figure 3). The increase in age at first R01 began to stabilize in the early 2000s, paralleling the stabilization of time from degree to first R01 award, as noted above.

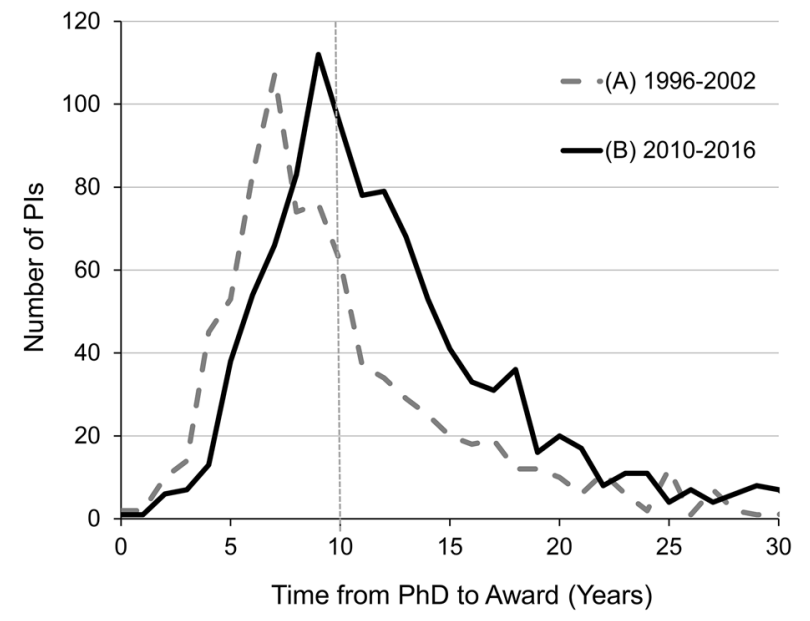

Figure 4. Time from PhD degree to first NCI R01 award for NIs with PhDs. Comparison of New Investigators from cohort A (awarded in FYs 1996-2002) to cohort B (awarded in FYs 2010-2016) shows an increase in time from PhD degree to first $\mathrm{NCl}$ R01-equivalent award. The vertical line indicates the 10 -year post-degree ESI designation.
Changing demographics and the NIH ESI policy. The NIH ESI policy, implemented in 2009, defined an ESI as an NI within 10 years of terminal research degree or clinical training, whichever was later. To assess the suitability of the 10-year ESI definition in the context of the NCI early-career investigator population, we analyzed the distribution of time from PhD to first R01 award for 2 cohorts of incoming PIs. The first cohort ("A") entered the NCI R01 workforce during FYs 1996-2002, prior to the implementation of the ESI policy, and the second cohort ("B") entered during FYs 2010-2016, following the implementation of the ESI policy. Comparing these cohorts illustrates that the proportion of NIs entering the NCI R01 PI workforce covered by the ESI definition has changed over the years: $65 \%$ of NIs from cohort A entered the NCI R01 workforce within 10 years of their $\mathrm{PhD}$, whereas only $46 \%$ of the NIs from cohort B were within 10 years of their $\mathrm{PhD}$ (Figure 4). Thus, the share of PhD NIs eligible to benefit from the ESI policy has declined.

Retention and attrition of NCI RO1 PIs. To detect any changes over time in retention of first-time R01 awardees in the NCI R01 workforce, we collected data for NIs in 5 different fiscal year cohorts, based on the year they received their first R01 award: 1992, 1997, 2002, 2007, and 2011. We followed these 5 cohorts through FY 2017 (Figure 5A). At the end of the first R01 budget period, typically 5 years after the first award, the percentage of PIs holding an NCI R01 award decreased substantially in all the cohorts, indicating an inability to either renew their original award or receive another new R01 award, or lack of application. Similar substantial decreases have been shown in other NIH analyses $(20,21)$. The percentage of PIs who held an NCI R01 six years after their first award was higher for earlier cohorts (41\% for the FY 1992 cohort, $42 \%$ for FY 1997, and $40 \%$ for FY 2002) than for later cohorts (26\% for FY 2007 and 34\% for FY 2011). Comparing the earlier versus later cohorts revealed a decrease in the overall proportion of NIs retained 6 years after the initial grant award.

To better understand the decreased retention, we analyzed the funding status of the most recent (FY 2011) cohort of NIs 6 years after the award was made, when funding from their initial award had ended and when an additional award would be needed to maintain their R01 funding (Figure 5B). Of this cohort, 34\% had an NCI R01 award in FY 2017 and 7\% had only a non-NCI NIH R01 award. Forty-five percent applied unsuccessfully for any NIH R01 funding during FYs 2012-2017, and 14\% did not apply at all during this time frame. Notably, $75 \%$ of the PIs who had R01 funding in FY 2017 received new (Type 1) awards, as opposed to competing renewals (Type 2) of their initial R01 awards.

Time in the NCI PI workforce since the first R01. Our observation that the number of PIs who are 65 or older has been steadily growing suggested that recent awardees had longer careers as Established Investigators (EIs) than PIs in the past. Thus, we examined all awarded PIs in a given fiscal year and determined how many 
A

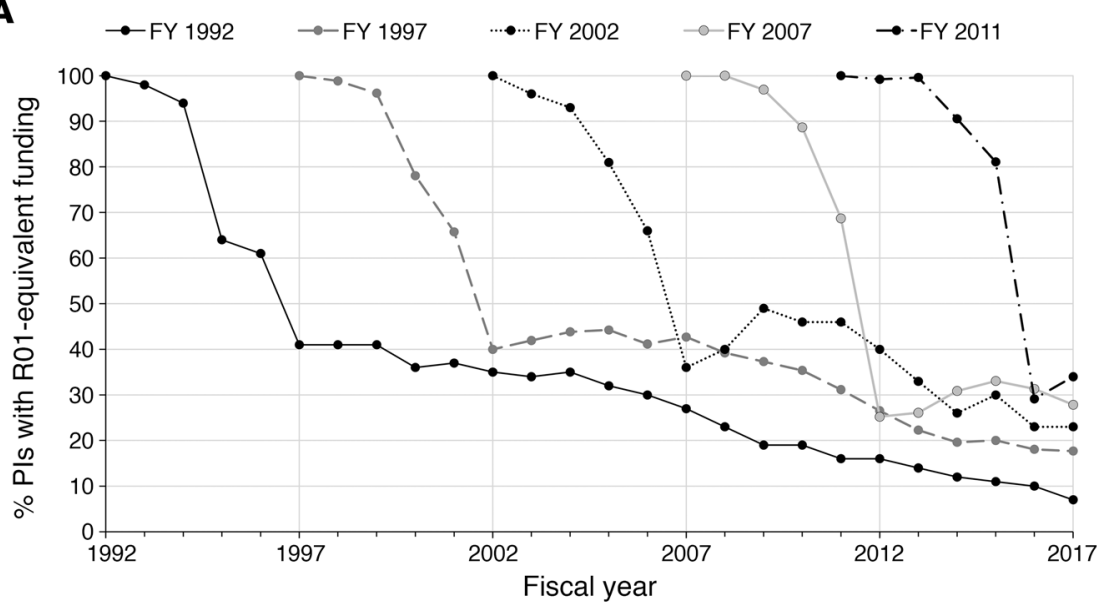

B

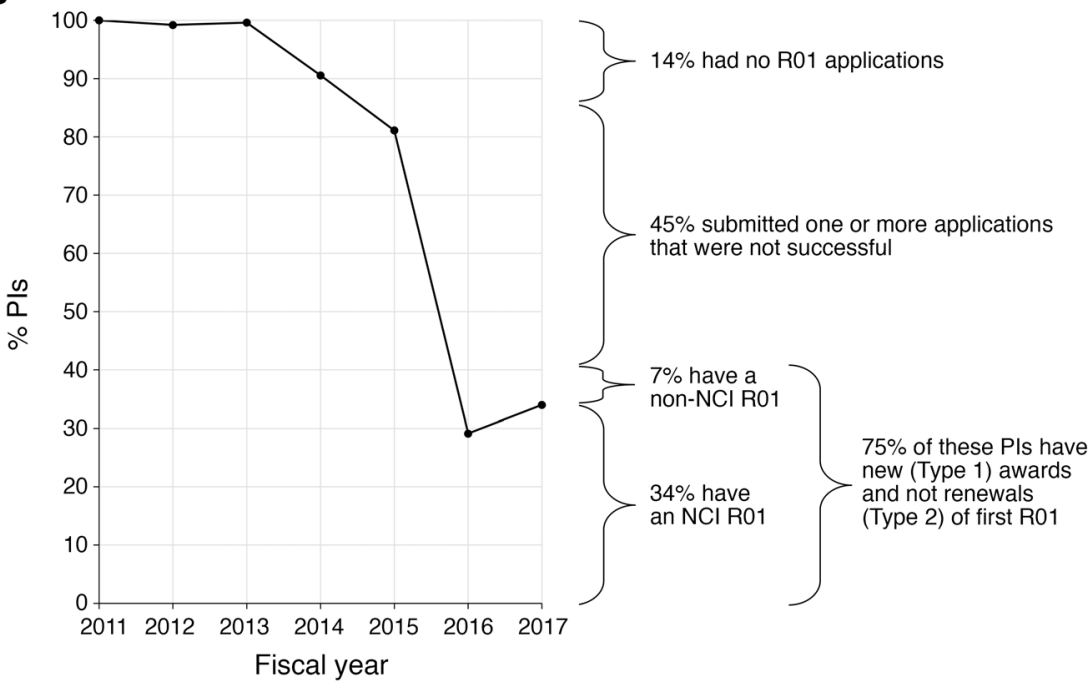

Figure 5. Percentage of PIs holding NCI R01-equivalent funding after their first R01-equivalent award. (A) Funding trend for New Investigators who received their first R01-equivalent funding in FYs 1992, 1997, 2002, 2007, and 2011. The successful funding of the cohort of investigators who received their first R01 equivalent in the indicated years is followed until FY 2017. Awards are limited to R01 equivalents from NCI. (B) Subsequent applications and awards for Pls who received their first NCI R01 award in FY 2011.

years had elapsed since their first R01 award. This duration of time may include gaps, that is, noncontinuous R01 funding. We plotted the cumulative distribution of PIs by number of years since their first R01 award for fiscal FYs 1990, 2000, 2010, and 2016 (Figure 6). The distribution shifts to the right over these 4 time points, suggesting that the PIs in more recent years have maintained substantial NCI research funding over a longer time span than PIs in earlier years. Interestingly, the most recent curves, corresponding to FY 2010 and FY 2016, are overlapping, suggesting a plateau in duration in the NCI R01-funded workforce.

\section{Discussion}

Here we report major changes in the demographics of the NCI R01-funded PI workforce from FY 1990 to FY 2016. The workforce has aged dramatically over this time frame as a result of a many-fold increase in older applicants, a decrease in young applicants, and a decrease in retention of New Investigators over time. In this discussion, we elucidate factors behind the aging and describe evidence-based strategies that the NCI is implementing to help early-career scientists, so as to maintain a robust pipeline and to sustain the cancer research workforce. It is difficult to determine the optimal age distribution of investigators. A diversity of ages may be beneficial to the research enterprise.

Mechanistically, the aging of the NCI R01 workforce was caused by dramatic increases in the number and proportion of older PIs applying for NCI R01 awards and concurrent decreases in the number and proportion of younger applicants. These diametrically opposed trends in applicant dynamics produced an older funded workforce. The aging was not due to differences in funding rate between older and younger PIs: rates were statistically similar between different age groups in any given fiscal year, indicating that younger PIs were as successful in competing for R01 awards as older PIs.

Several interrelated factors influenced why fewer younger PIs applied and why more older PIs applied over the examined time frame. The decrease in number of younger PIs applying for NCI R01 awards is largely due to factors outside of the NCI's institutional control, such as changes in career stage timing and the repeal of the mandatory retirement age. With respect to career stage, the increased time from degree to first R01 application and to first R01 award indicates that NIs may now be spending longer times in activities such as postdoctoral training, academic job search, and lab startup than NIs in the 1990s. The increased time in such pre-application activities is directly increasing the age at first R01 application and award. Another consequence of the increased time spent during early career stages is that fewer researchers are achieving PI positions at young ages. Thus, fewer young PIs are applying for and receiving R01 awards. The NCI and the NIH have little or no influence over factors affecting age and career stage timing such as length of postdoctoral fellowships, participation in multiple fellowships, and availability of tenure track positions. In order to achieve decreases in age or training times, collaboration with and commitment of extramural institutions will be necessary (22).

The repeal of the mandatory retirement age in 1994 meant that PIs who entered the system early and stayed in the system could have longer careers and thus extend their research productivity. The increased number of older R01 PIs over time may have also contributed indirectly to the decrease in the number of young applicants: as older PIs stayed in the workforce longer, the number of jobs available for early-career PIs was reduced, which, in turn, may have delayed achievement of some career milestones $(7,22,23)$. 


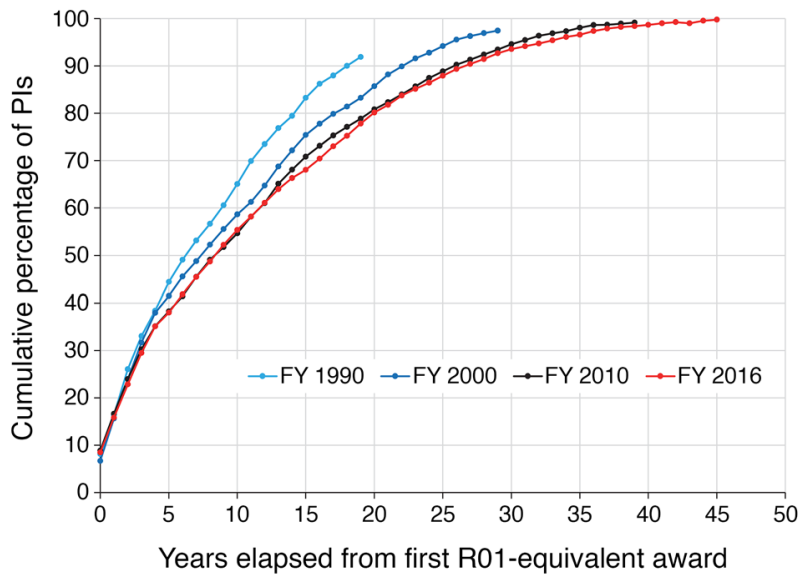

Figure 6. Cumulative percentage of NCI Principal Investigators holding R01-equivalent grants in FYs 1990, 2000, 2010, and 2016. Pls that were awarded R01-equivalent grants are plotted versus the time since their first award. Individuals are counted only once even when they hold multiple R01-equivalent awards or are funded through multiple-PI (MPI) grants. The date of first award prior to FY 1970 is not available; thus not all curves reach the $100 \%$ mark.

The doubling of the NIH budget during FYs 1998-2003 appears, paradoxically, to have contributed to the substantial increase in the number of older applicants. During this budget doubling, the number and proportion of NCI R01 awardees aged 45 through 64 increased dramatically, whereas the proportion of younger awardees shrank. Thus, we conclude that the NIH doubling preferentially benefited NCI PIs who already had independent research positions, as opposed to giving a boost to earlycareer scientists (24). Later events that may have also influenced the evolution of the NCI R01 workforce include the American Recovery and Reinvestment Act in FYs 2009-2010 and the fiscal austerity policy of sequestration in FY 2013.

Although the implementation of the NIH ESI policy appears to have stopped a further increase in time to first R01, the 10-year post-degree time frame defining ESI eligibility may already be outdated. It may no longer be adequate for benefiting as many current early-career scientists as originally planned. Our comparison of NI cohorts before and after the implementation of the NIH ESI policy indicates that a substantially higher percentage of NIs in the post-implementation cohort missed the ESI cutoff. Thus, a 10-year time frame post-degree may be too short for earlycareer cancer researchers who are training longer, setting up labs, or tending to family responsibilities. Likewise, career development (K) awardees, who typically have a 5-year mentored grant, are at risk of missing the 10-year ESI cutoff. Instead of being rewarded for enhancing their career by applying for and receiving $\mathrm{K}$ awards, they run the risk of missing a critically important career-boosting opportunity. Based on the data presented here, an extension of the ESI definition to 12 to 13 years post-degree would give current NIs an advantage equivalent to that given to NIs in the early 2000s. Although this measure may increase the age at first R01 award, it would address the demographic shift and help meritorious early-career researchers join the workforce.

The results of our analyses point to increasing difficulty, relative to the past, for young and early-career PIs to join and remain in the NCI-funded workforce. The decrease in retention of recent cohorts compared with earlier cohorts highlights the challenge in sustaining a career in cancer research. Later cohorts experienced a less favorable funding climate and increased competition for funding for subsequent awards. For example, the FY 1997 cohort of NIs completed its first budget period in 2002, during the time of the doubling of the NIH budget, when the success rate of NCI R01 applications was 26\%, which is double the recent FY 2016 success rate of $13 \%$.

To help early-career cancer researchers launch and sustain their scientific careers, the NCI has recently implemented new policies that began after the period of this analysis. To help launch careers of ESIs, in FY 2018 the NCI increased awards to ESIs by more than $25 \%$ compared with FY 2017. The NCI has also used "select pay" - the funding of grants outside the payline - to improve success rates for ESIs in recent years. As younger PIs tend to be more diverse in terms of race, ethnicity, and gender, the NCI views this extra support for ESIs as a key component of its plan to increase overall diversity of its scientific workforce. In addition to increasing ESI success rates, starting in FY 2018 the NCI also began converting all ESI R01 awards scoring within the Established Investigator payline (e.g., 9th percentile in 2019) into ESI R37 Method to Extend Research in Time (MERIT) awards (25), which provide up to 2 extra years of funding. This additional time should enable PIs to make more research progress, to compete for a second project before recompeting their first award, to take more risks, and to innovate. Importantly, the 2 extra years may help these PIs sustain NCI R01 funding, and thus increase the proportion of PIs who remain in the NCI-funded workforce.

A better understanding of the NCI grant funding system may help early-career applicants secure and sustain funding. Specifically, competing renewal (Type 2) applications have a higher success rate than new (Type 1) applications at the NCI (26). Yet, as our results showed, the most common way that FY 2011 NIs sustained their R01 funding was by competing for a new award, instead of by competing for a renewal of their first R01 award. Our data suggest that a better strategy for sustaining R01 funding, which should be communicated to young investigators, may be to apply both for a second project during the initial project period and for a renewal.

The NCI is increasing communication about its data and application processes to better disseminate information that might not be common knowledge. For example, the NCI recently launched a grantee-focused blog that covers topics such as the NCI's fiscal landscape, funding decisions, grant policy news, and processes (see https://www.cancer.gov/grants-training/nci-bottom-line-blog). The NCI will continue to analyze its programs and processes to develop data-informed policies that sustain, invigorate, and diversify its workforce of cancer researchers.

\section{Methods}

Source data. Data on NIH extramural applications, grants, and PIs were retrieved during 2017-2019 from the NIH Information for Management, Planning, Analysis and Coordination II (IMPACII) database through NIH internal electronic interfaces or from the Division of Statistical Analysis and Reporting in the NIH Office of Extramural 
Research. Predecisional and sensitive data are reported in aggregate and only shown if the aggregated total in any group is $\geq 12$.

Analysis. Analysis and visualizations were performed with Excel, Excel VBA, R, and Python. Funding rates of different age groups were compared using the Python chi-squared contingency function in SciPy (version 1.2.1).

Definitions. For investigator populations and other funding-related terms, we use the NIH definitions, which are available from the NIH glossary (https://grants.nih.gov/grants/glossary.htm) and described in Table 1. Terms describing the career stages of investigator populations (NI, ESI, EI) are portrayed in Figure 1.

Analytical decisions and additional definitions. We define 9 age groups, 7 of which are 5-year age groups encompassing PIs aged 35-70. The two additional groups are PIs younger than 35 and PIs older than 70. We track the numbers and proportions for these 9 groups from FY 1990 through FY 2016. We focus on this time frame for 2 reasons: (a) data available prior to FY 1990 are less complete, and (b) FY 2016 was the last complete fiscal year prior to the NGRI, which was implemented in the middle of FY 2017. Thus, bounding the analysis at FY 2016 avoids confounding of data by the implementation of the NGRI.

We count and define "unique PI" applicants and awardees as PIs on any R01 application, whether a single-PI application or a multiple-PI application, in a given fiscal year. Each PI is counted only once per year regardless of the number of applications or awards.

\section{Author contributions}

MDA conceptualized the project, designed and interpreted the analyses, and wrote the manuscript. RG conducted and interpreted the analyses and wrote the manuscript. AK conducted analyses and revised the manuscript. GFL designed and conducted analyses and revised the manuscript. ENB conducted analyses. JGC designed analyses and revised the manuscript. LMB conceptualized the project, interpreted the analyses, and wrote the manuscript.

\section{Acknowledgments}

We thank Ed Harlow for helpful discussions and feedback and insight on the analyses and the manuscript. We thank Diane Palmieri for her insightful comments on the manuscript.

Address correspondence to: Melissa D. Antman, Center for Research Strategy, National Cancer Institute, Building 31A 10A10B, 31 Center Drive, Bethesda, Maryland 20892, USA. Phone: 240.781.3441; Email: melissa.antman@nih.gov.

AK's present address is: Division of Cancer Control and Population Sciences, National Cancer Institute, NIH, Rockville, Maryland, USA. ENB's present address is: Office of Research on Women's Health; Division of Program Coordination, Planning and Strategic Initiatives; Office of the Director; NIH, Rockville, Maryland, USA. JGC's present address is: Independent Consultant, Olney, Maryland, USA.
1. National Cancer Institute. National Cancer Institute Overview and Mission. https://www.cancer. gov/about-nci/overview. Updated April 6, 2018. Accessed February 10, 2021.

2. Alexander H, Liu QC. The aging of full-time U.S. medical school faculty: 1967-2007. AAMC Analysis in Brief. 2009;9(4):1-2.

3. Kaiser J. Biomedical Research. The graying of NIH research. Science. 2008;322(5903):848-849.

4. Kaiser J. Biomedical Research. A call for NIH youth movement. Science. 2014;346(6206):150-151.

5. Liu C, Alexander H. The changing demographics of full-time medical school faculty: 1966-2009. AAMC Analysis in Brief. 2011;11(8):1-2.

6. Matthews KR, et al. The aging of biomedical research in the United States. PLoS One. 2011;6(12):e29738.

7. NIH Advisory Committee to the Director. Biomedical Research Workforce Working Group Report. https://www.acd.od.nih.gov/documents/ reports/Biomedical_research_wgreport.pdf. Accessed November 12, 2020.

8. Heggeness ML, et al. Policy implications of aging in the NIH-funded workforce. Cell Stem Cell. 2016;19(1):15-18.

9. NIH Office of Extramural Research. Average age and degree of NIH R01-equivalent investigators Table 440-16. grants.nih.gov/grants/new_investigators/Age_Degree-First-Time-117-16_RFM_ 1ls_25march2016_DR-Approved.xlsx. Accessed November 12, 2020.

10. Rockey S. Age distribution of NIH principal investigators and medical school faculty. Extramural Nexus blog. February 13, 2013. https://nexus.od.nih.gov/all/2012/02/13/ age-distribution-of-nih-principal-investiga- tors-and-medical-school-faculty/. Accessed November 12, 2020.

11. Blau DM, Weinberg BA. Why the US science and engineering workforce is aging rapidly. Proc Natl Acad Sci U S A. 2017;114(15):3879-3884.

12. Levitt M, Levitt JM. Future of fundamental discovery in US biomedical research. Proc Natl Acad Sci U S A . 2017;114(25):6498-6503.

13. 21st Century Cures Act, Public Law 114-255, 114th Congress (2016). https://www.congress. gov/114/plaws/publ255/PLAW-114publ255.htm. Accessed November 12, 2020.

14. Alberts B, et al. Improving support for young biomedical scientists. Science. 2018;360(6390):716-718.

15. Charette MF, et al. Shifting demographics among research project grant awardees at the National Heart, Lung, and Blood Institute (NHLBI). PLoS One. 2016;11(12):e0168511.

16. Lauer M. Mid-career investigators and shifting demographics of NIH grant recipients. Extramural Nexus blog. March 6, 2017. https://nexus. od.nih.gov/all/2017/03/06/mid-career-investigators-shifting-demographics-nih-grant-recipients/. Accessed February 10, 2021.

17. Nikaj S, et al. Examining trends in the diversity of the U.S. National Institutes of Health participating and funded workforce. FASEB J. 2018;32(12):6410-6422.

18. Tabak L. Enhancing stewardship: the Next Generation of Researchers Initiative. Presented at: 9 th Joint Meeting of the NCI Board of Scientific Advisors and the National Cancer Advisory Board; June 19-21, 2017; Bethesda, Maryland, USA. https://deainfo.nci.nih.gov/ advisory/joint/0617/Tabak.pdf. Accessed
November 12, 2020.

19. Walsh R, et al. An evaluation of the National Institutes of Health Early Stage Investigator policy: using existing data to evaluate federal policy. Res Eval. 2018;27(4):380-387.

20. Haggerty PA, Fenton MJ. Outcomes of early NIH-funded investigators: experience of the National Institute of Allergy and Infectious Diseases. PLoS One. 2018;13(9):e0199648.

21. Rockey S. Retention rates for first-time R01 awardees. Extramural Nexus blog. October 28, 2014. https://nexus.od.nih.gov/all/2014/10/28/ retention-of-first-time-rO1-awardees/. Accessed November 12, 2020.

22. National Academies of Sciences Engineering Medicine, et al. The Next Generation of Biomedical and Behavioral Sciences Researchers: Breaking Through. National Academies Press; 2018.

23. Mason JL, et al. Labor and skills gap analysis of the biomedical research workforce. FASEB J. 2016;30(8):2673-2683.

24. National Research Council of the National Academies (US). Bridges to Independence: Fostering the Independence of New Investigators in Biomedical Research. National Academies Press; 2005.

25. NIH Guide for Grants and Contracts. NCI Method to Extend Research in Time (MERIT) Award for Early Stage Investigators (R37). Notice NOT-CA-18-037. https://grants. nih.gov/grants/guide/notice-files/NOTCA-18-037.html. Updated January 23, 2018. Accessed November 12, 2020.

26. NIH RePORT Data Book. Success Rates: R01-Equivalent and Research Project Grants. https://report.nih.gov/nihdatabook/category/10. Accessed November 12, 2020. 